\title{
A new null diagnostic customized for reconstructing the properties of dark energy from BAO data
}

\author{
Arman Shafieloo $^{a, b, c}$, Varun Sahni $^{d}$, and Alexei A. Starobinsky ${ }^{e, f}$ \\ a Asia Pacific Center for Theoretical Physics, Pohang, Gyeongbuk 790-784, Korea \\ ${ }^{b}$ Department of Physics, POSTECH, Pohang, Gyeongbuk 790-784, Korea \\ ${ }^{c}$ Institute for the Early Universe WCU, Ehwa Womans University, Seoul, 120-720, Korea \\ ${ }^{d}$ Inter-University Centre for Astronomy and Astrophysics, Post Bag 4, Ganeshkhind, Pune 411 00\%, India \\ ${ }^{e}$ Landau Institute for Theoretical Physics RAS, Moscow 119334, Russia and \\ ${ }^{f}$ Research Center for the Early Universe (RESCEU), \\ Graduate School of Science, The University of Tokyo, Tokyo 113-0033, Japan
}

\begin{abstract}
Baryon Acoustic Oscillations (BAO) provide an important standard ruler which can be used to probe the recent expansion history of our universe. We show how a simple extension of the $O m$ diagnostic, which we call $O m 3$, can combine standard ruler information from BAO with standard candle information from type Ia supernovae (SNIa) to yield a powerful novel null diagnostic of the cosmological constant hypothesis. A unique feature of $\mathrm{Om} 3$ is that it requires minimal cosmological assumptions since its determination does not rely upon prior knowledge of either the current value of the matter density, $\Omega_{0 m}$ and the Hubble constant $H_{0}$, or the distance to the last scattering surface. Observational uncertainties in these quantities therefore do not affect the reconstruction of $\mathrm{Om} 3$. We reconstruct Om3 using the Union 2.1 SNIa data set and BAO data from SDSS, WiggleZ and 6dFGS. Our results are consistent with dark energy being the cosmological constant. We show how $\mathrm{Om}$ and $\mathrm{Om} 3$ can be used to obtain accurate model independent constraints on the properties of dark energy from future data sets such as BigBOSS.
\end{abstract}

\section{INTRODUCTION}

The expansion of the universe appears to have undergone a dramatic change in its recent past with most observations suggestive of the fact that cosmic expansion began to accelerate when the scale factor $a(t)$ of the Universe was about 0.6 of its present value, i.e. at the redshift $z \sim 0.7$. Although cosmic acceleration is a dramatic phenomenon, evidence for it is indirect and stems from data observed along our past light cone and involving either standard candles (SNIa) or standard rulers $(\mathrm{BAO}, \mathrm{CMB})[\underline{1}[\underline{6}]$.

The raison d'etre behind cosmic acceleration remains unknown and the fact that it might signal the need for new physics is partly responsible for the high levels of activity marking this field. Possible drivers of acceleration range from the Einstein's early suggestion of the cosmological constant [7] to more elaborate constructions collectively called Dark Energy (DE) which may equally well be based on the introduction of a new physical field (physical DE) or on modifying the Einstein General Relativity (GR) (geometrical DE), or both [8, 9]. To distinguish between these widely varying alternatives, model independent diagnostic tools capable of differentiating different classes of DE models are of great help [9].

With this in mind, we introduced the $O m$ diagnostic [10, 11] which successfully distinguishes evolving DE from the cosmological constant on the basis of observations of the expansion history $H(z)$. The two-point $O m$ diagnostic can be defined as follows

$$
O m\left(z_{2} ; z_{1}\right)=\frac{h^{2}\left(z_{2}\right)-h^{2}\left(z_{1}\right)}{\left(1+z_{2}\right)^{3}-\left(1+z_{1}\right)^{3}}, \quad h(z)=H(z) / H_{0}
$$

so that [10]

$$
O m(z ; 0) \equiv O m(z)=\frac{h^{2}(z)-1}{(1+z)^{3}-1} .
$$

For a spatially flat universe, $H(z)$ can be recovered from the luminosity distance via a single differentiation [12 16]

$$
H(z)=\left[\frac{d}{d z}\left(\frac{D_{L}(z)}{1+z}\right)\right]^{-1},
$$


which makes $H(z)$ more robustly determined than the equation of state

$$
w(x)=\frac{(2 x / 3) d \ln H / d x-1}{1-\left(H_{0} / H\right)^{2} \Omega_{0 m} x^{3}}, \quad x=1+z,
$$

which involves knowing the second derivative of $D_{L}$ as well as the cosmological matter density $\Omega_{0 m}$. Thus observational uncertainties in $D_{L}$ and $\Omega_{0 m}$ muddy the reconstruction of $w(z)$ to a much greater extent than $H(z)$ and $O m(z)$ 10, 11]. The expansion history, $H(z)$, can also be determined directly from ages of passively evolving galaxies [17], the time drift of the cosmological redshift [18], radial BAO's [19 21] or from a combination of the Alcock-Paczynski test and galaxy redshift space distortions [22]. This opens up the possibility of determining $O m(z)$ from a combination of data sets involving the low redshift universe $(z \lesssim$ few $)$, precisely where cosmic acceleration seems to have originated !

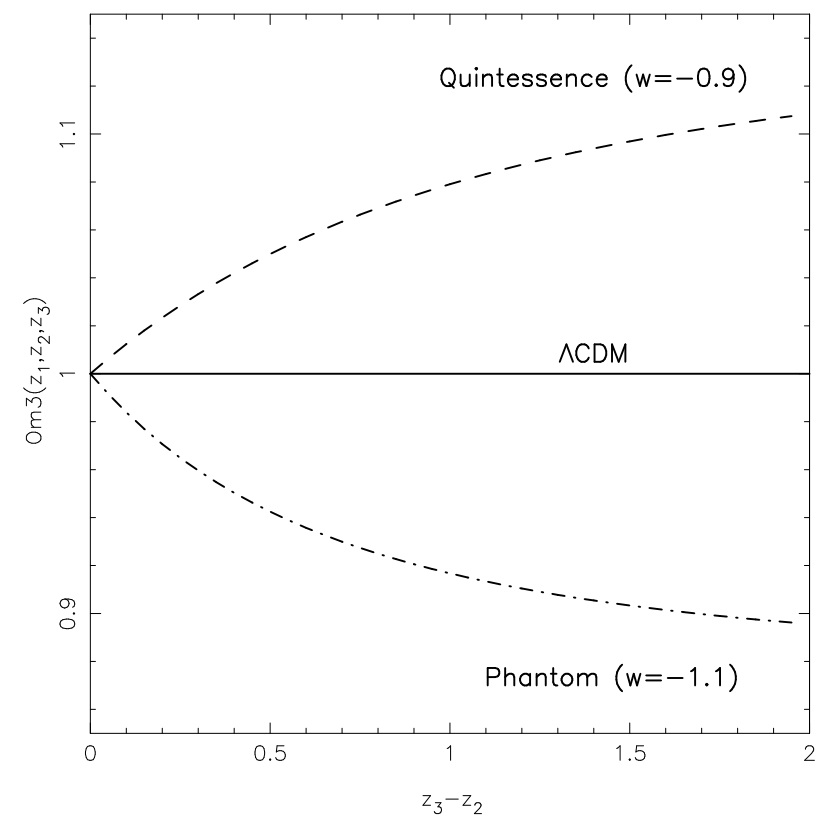

FIG. 1: The Om3 diagnostic (1.9) is shown as a function of separation, $z_{3}-z_{2}$, for $z_{1}=0.2, z_{2}=0.35$ and $\Omega_{0 m}=0.27$. This diagnostic is clearly able to discriminate quite well between evolving DE models and $\Lambda$ CDM, since, for $\Lambda$ CDM the value of Om3 stays pegged at unity, while for Quintessence/Phantom Om3 rapidly evolves to larger/smaller values as the separation $z_{3}-z_{2}$ increases.

The possibility of using $\mathrm{Om}$ as a null diagnostic follows from the fact that, for the cosmological constant

$$
O m\left(z_{2} ; z_{1}\right)=\Omega_{0 m}
$$

In other words, the value of $\mathrm{Om}$ is redshift independent for the cosmological constant, while for other models of cosmic acceleration $O m(z)$ is redshift dependent. This important property of $O m$ can be used to considerable advantage by defining the difference diagnostic [10]

$$
O m_{\text {diff }}\left(z_{1}, z_{2}\right):=O m\left(z_{1}\right)-O m\left(z_{2}\right)
$$

and the ratio diagnostic

$$
O m_{\text {ratio }}\left(z_{1}, z_{2}, z_{3}, z_{4}\right):=\frac{O m\left(z_{2} ; z_{1}\right)}{O m\left(z_{4} ; z_{3}\right)}
$$

From (1.5) one immediately finds that the following equalities must hold for the cosmological constant

$$
O m_{\text {diff }}\left(z_{1}, z_{2}\right)=0, \quad O m_{\text {ratio }}\left(z_{1}, z_{2}, z_{3}, z_{4}\right)=1
$$

A departure of $O m_{\text {ratio }}$ from unity therefore serves as a 'smoking gun' or null test for concordance cosmology: $\Lambda$ CDM. 
If any two of the four redshifts in $O m_{\text {ratio }}$ are identical then $O m_{\text {ratio }}$ reduces to the 3 point diagnostic Om3

$$
O m_{\text {ratio }}\left(z_{1}, z_{2}, z_{1}, z_{3}\right):=\operatorname{Om} 3\left(z_{1}, z_{2}, z_{3}\right)=\frac{O m\left(z_{2} ; z_{1}\right)}{O m\left(z_{3} ; z_{1}\right)}
$$

where $O m 3=1$ for $\Lambda \mathrm{CDM}^{1}{ }^{1}$ (See [27, 29, [30] for other discussions of the $O m$ diagnostic.)

In this paper we focus on $\mathrm{Om} 3$ and show that the main difference between $\mathrm{Om} 3$ and $\mathrm{Om}$ is that these two diagnostics test the cosmological constant hypothesis using different cosmological observables. Whereas $\mathrm{Om}$ is a powerful null diagnostic of the cosmological constant as it requires only a prior knowledge of the expansion history, $h(z)$, one should note that deriving $h(z)$ directly from cosmological observables in a purely model independent and non-parametric manner is not always a simple task. Although supernovae data do allow one to reconstruct $h(z)$ in a model independent manner [25], deriving $h(z)$ directly from large scale structure data seems to be considerably more difficult (see [27] for detailed discussions). In contrast to $\mathrm{Om}$, the $\mathrm{Om} 3$ diagnostic is specifically tailored to be applied directly on baryon acoustic oscillation data, and depends on fewer cosmological observables than $\mathrm{Om}$ in this case.

Combining BAO information from the SDSS, WiggleZ \& 6dF surveys with SNIa information from the Union2.1 data set, allows us to determine the three point diagnostic, $O m 3$, at several independent redshift bins thereby placing robust constraints on the nature of dark energy.

\section{THE OM3 DIAGNOSTIC RECONSTRUCTED FROM SNIA AND BAO DATA}

A key role in the determination of Baryon Acoustic Oscillations is played by the 'dilation-scale' distance [33]

$$
D_{V}(z)=\left[D(z)^{2} \frac{c z}{H(z)}\right]^{1 / 3}, \quad \text { where } D(z):=\frac{D_{L}(z)}{1+z}=\int \frac{c d z}{H(z)}
$$

and we have used $D_{L}(z)=(1+z)^{2} D_{A}(z)$ to relate the luminosity distance $D_{L}$ to the angular size distance $D_{A}$ and also made the assumption that the universe is spatially flat.

Other important parameters which can be extracted from BAO's include the acoustic parameter

$$
A(z)=\frac{100 D_{V}(z) \sqrt{\Omega_{0 m} h^{2}}}{c z}
$$

and the ratio

$$
d(z)=\frac{r_{s}\left(z_{\mathrm{CMB}}\right)}{D_{V}(z)}
$$

where $r_{s}\left(z_{\mathrm{CMB}}\right)$ is the sound horizon at the epoch when CMB photons decouple from baryons.

Equation (2.1) allows us to relate the expansion history, $H(z)$, to $D_{V}$ and $D$ as follows

$$
H(z)=\frac{c z D(z)^{2}}{D_{V}(z)^{3}}
$$

Independent measurements of $D_{V}$ and $D_{L}$ can therefore be used to reconstruct $H(z)$, as discussed in [27].

In this paper we shall demonstrate that the ratio of the Hubble parameter at two redshifts can play a key role in cosmological reconstruction. To see this note that

$$
H\left(z_{i} ; z_{j}\right):=\frac{H\left(z_{i}\right)}{H\left(z_{j}\right)}=\frac{z_{i}}{z_{j}}\left[\frac{D\left(z_{i}\right)}{D\left(z_{j}\right)}\right]^{2}\left[\frac{D_{V}\left(z_{j}\right)}{D_{V}\left(z_{i}\right)}\right]^{3}
$$

[1] Note a passing similarity between the diagnostic pair $\left\{O m_{\text {ratio }}, O m_{\text {diff }}\right\}$ and the Statefinder diagnostic [23] $\{r, s\}$, where $r=\dddot{a} / a H^{3}$, $s=(r-1) / 3(q-1 / 2)$. In both cases, for the $\Lambda$ CDM model one finds $\left\{O m_{\text {ratio }}, O m_{\text {diff }}\right\}=\{1,0\}$ and $\{r, s\}=\{1,0\}$. However, unlike the statefinder pair, the values of $O m_{\text {diff }} \& O m_{\text {ratio }}$ are strongly correlated, as seen from (1.6) and (1.9). Additionally, neither of $O m_{\text {diff }}, O m_{\text {ratio }}$ is able to distinguish steady state cosmology (SS) $\left(\Omega_{\Lambda}=1, \Omega_{0 m}=0\right)$, from SCDM $\left(\Omega_{\Lambda}=0, \Omega_{0 m}=1\right)$ while the $O m$ diagnostic and the Statefinders are more successful on this score, since $\operatorname{Om}(z)=0$ for SS while $\operatorname{Om}(z)=1$ for SCDM; $\operatorname{similarly}$ $\{r, s\}=\{1,0\}$ for SS while $\{r, s\}=\{1,1\}$ for SCDM (see also 24$]$ ). 
equivalently

$$
H\left(z_{i} ; z_{j}\right)=\left(\frac{z_{j}}{z_{i}}\right)^{2}\left[\frac{D\left(z_{i}\right)}{D\left(z_{j}\right)}\right]^{2}\left[\frac{A\left(z_{j}\right)}{A\left(z_{i}\right)}\right]^{3}=\frac{z_{i}}{z_{j}}\left[\frac{D\left(z_{i}\right)}{D\left(z_{j}\right)}\right]^{2}\left[\frac{d\left(z_{i}\right)}{d\left(z_{j}\right)}\right]^{3},
$$

in other words, ratio's of BAO parameters $D_{V}, A, d(z)$ are related to the ratio of the Hubble parameter. From (2.6) we find that $H\left(z_{i} ; z_{j}\right)$ does not depend either on $H_{0}, \sqrt{\Omega_{0 m} h^{2}}$, or even the CMB parameter $r_{s}\left(z_{\mathrm{CMB}}\right)$.

We therefore arrive at the following important result: the value of $D_{L}$ together with the value of either of the BAO paramaters $\left\{D_{V}, A, d\right\}$, determined at three independent redshifts, is sufficient to evaluate the Om3 diagnostic and define a null test of the cosmological constant hypothesis!

This follows immediately from (1.1) \& (1.9), since, from the definition of $O m 3$ in (1.9) it follows that

$$
\operatorname{Om} 3\left(z_{1} ; z_{2} ; z_{3}\right)=\frac{H\left(z_{2} ; z_{1}\right)^{2}-1}{x_{2}^{3}-x_{1}^{3}} / \frac{H\left(z_{3} ; z_{1}\right)^{2}-1}{x_{3}^{3}-x_{1}^{3}}, \text { where } x=1+z,
$$

where $H\left(z_{i} ; z_{j}\right)$ is determined using (2.5) or (2.6). We should note that interchanging redshifts allows us to define different variants of $\mathrm{Om} 3\left(z_{1}, z_{2}\right.$ and $z_{3}$ should not be necessarily sorted in redshift). Large galaxy redshift survey's including SDSS, WiggleZ and 6dFGS have determined the BAO parameters at several distinct redshift points [3 [6]. In this paper we shall utilize these results to reconstruct $\mathrm{Om} 3$, thereby obtaining useful constraints on the nature of dark energy. It should be stressed that the model independent method which we advocate does not rely on a knowledge of either the matter density $\Omega_{0 m}$ or the Hubble parameter $H_{0}$. Its scope and prowess is therefore likely to dramatically improve as new data is added to the burgeoning BAO inventory by dedicated upcoming surveys such as BOSS [36], BigBOSS [37], J-PAS [38] and Euclid [39].

\section{A. Cosmological Data Sets}

As we stressed above, in order to determine $O m 3$ one only needs to know the luminosity distance, $D_{L}\left(z_{i}\right)$, and any one of the BAO parameters $D_{V}\left(z_{i}\right), A\left(z_{i}\right), d\left(z_{i}\right)$. In this paper we shall use the Union 2.1 supernova data set [2] to reconstruct $D_{L}$, and the SDSS DR7 [3], WiggleZ [4, 5] and 6dFGS [6] determinations of the BAO parameters to determine $O m 3$. Below we briefly describe these data sets and our method of extracting $O m 3$ from them.

1. Union 2.1 SNIa data. The Union 2.1 set [2] consists of 580 type Ia supernovae sampling the redshift range $0.015 \leq z \leq 1.414$. This data set includes 175 SNIa at low redshifts $z<0.1$.

Union 2.1 supernovae allow us to determine the luminosity distance $D_{L}(z)$ in a model independent manner following the efficient smoothing ansatz proposed in [25]. Namely, a smoothed value for the luminosity distance, $D_{L}^{S}(z)$, is constructed from the fluctuating 'raw' value implied by data, $D_{L}(z)$, by smoothing the latter using a low pass filter $F$ having a smoothing scale $\Delta$

$$
D_{L}^{S}(z)=\int D_{L}\left(z^{\prime}\right) F\left(\left|z-z^{\prime}\right| ; \Delta\right) d z^{\prime}
$$

where $F\left(\left|z-z^{\prime}\right| ; \Delta\right)$ is a variant of the Gaussian filter $F_{\mathrm{G}} \propto \exp \left(-\left|\mathbf{x}-\mathbf{x}^{\prime}\right|^{2} / 2 \Delta^{2}\right)$. Specifically, one follows the iterative procedure [25]

$$
\begin{gathered}
\ln D_{L}^{S}(z, \Delta)=\ln D_{L}^{g}(z)+N(z) \sum_{i}\left[\ln D_{L}\left(z_{i}\right)-\ln D_{L}^{g}\left(z_{i}\right)\right] \times \exp \left[-\frac{\ln ^{2}\left(\frac{1+z_{i}}{1+z}\right)}{2 \Delta^{2}}\right] \\
N(z)^{-1}=\sum_{i} \exp \left[-\frac{\ln ^{2}\left(\frac{1+z_{i}}{1+z}\right)}{2 \Delta^{2}}\right]
\end{gathered}
$$

where $D_{L}^{g}(z)$ is a 'guessed' background model which is subtracted from the data before smoothing, thereby ensuring that it is the noise that is smoothed and not the luminosity distance! The background model is upgraded at each iteration step via $D_{L}^{g}(z) \rightarrow D_{L}^{S}(z)$ and it is found that convergence is reached within a few steps, and that cosmological reconstruction is quite insensitive to the initial guess value $D_{L}^{g}(z)$, which is reassuring. One might note that cosmological reconstruction using the smoothing ansatz usually yields a better fit (improved $\chi^{2}$ ) when compared with reconstruction using parametric methods [27]. The sensitivity of this method can be 
TABLE I: BAO distances, from [5]

\begin{tabular}{c|c|c|c}
\hline BAO sample & $z$ & $d(z)$ & $A(z)$ \\
\hline 6dFGS & 0.106 & $0.336 \pm 0.015$ & $0.526 \pm 0.028$ \\
SDSS & 0.2 & $0.1905 \pm 0.0061$ & $0.488 \pm 0.016$ \\
SDSS & 0.35 & $0.1097 \pm 0.0036$ & $0.484 \pm 0.016$ \\
WiggleZ & 0.44 & $0.0916 \pm 0.0071$ & $0.474 \pm 0.034$ \\
WiggleZ & 0.6 & $0.0726 \pm 0.0034$ & $0.442 \pm 0.020$ \\
WiggleZ & 0.73 & $0.0592 \pm 0.0032$ & $0.424 \pm 0.021$ \\
\hline
\end{tabular}

further enhanced by making the reconstruction error-sensitive via the substitution $\left[\ln D_{L}\left(z_{i}\right)-\ln D_{L}^{g}\left(z_{i}\right)\right] \rightarrow$ $\left[\ln D_{L}\left(z_{i}\right)-\ln D_{L}^{g}\left(z_{i}\right)\right] / \sigma_{D_{L}\left(z_{i}\right)}^{2}$ in (2.9); see [25 27] for more details, and [22, 27, 28] for other applications of the smoothing method.

\section{Data from Baryon Acoustic Oscillations}

Baryon acoustic oscillations - a relic of the pre-recombination universe - have been measured at 6 redshifts: $z=0.106,0.2,0.35,0.44,0.6$ and 0.73 . At these redshifts, the 'distilled BAO parameters' $A(z)$ and $d(z)$ have been determined to good accuracy, with the BAO detection itself being at the level of $2-3 \sigma$. Table 1 , which has been reproduced from [5], summarizes the BAO dataset.

Not all of the BAO data in Table 1 is statistically independent. As pointed out in [5], measurements belonging to the following redshift pairs are correlated: $z=(0.2,0.35), z=(0.44,0.6), z=(0.6,0.73)$, the correlation coefficient being $0.337,0.369$ and 0.438 , respectively. By using a BAO parameter associated with one of the two redshifts belonging to a correlated pair, one has 3 relatively independent parameters in all, which is precisely the correct number to determine Om3!

In this paper we use $d(z)$ results from Table 1 associated with the following redshift triplets to determine $O m 3$ : $(0.106,0.35,0.6),(0.2,0.44,0.73)$ and $(0.106,0.35,0.73)$. Our main result, therefore, consists of finding three independently obtained values for the $\mathrm{Om} 3$ diagnostic constructed from (2.7) \& (2.6) and using the BAO data in Table 1 jointly with Union 2.1 SNIa data.

\section{B. Results}

In figure 2 we show results for two variants of $O m 3$ using three uncorrelated measurements of the WiggleZ baryon acoustic oscillation survey. In the left column the results for the $\left(z_{1} ; z_{2} ; z_{3}\right)$ variant of $\mathrm{Om} 3$ are presented, while the right columns shows results for the $\left(z_{2} ; z_{3} ; z_{1}\right)$ variant. The spatially flat $\Lambda \mathrm{CDM}$ model $(\mathrm{Om} 3=1$ for all variants) is in good accord with the data. However, the quality of the data is not yet good enough to allow us to confront different cosmological models with data in a precise and, at the same time, model independent way. Propagation of errors results in large error bars on Om3 making it difficult to distinguish DE models from each other. It therefore appears that $O m 3$ has the potential to be used as a model independent future probe of cosmological models when the quality of data improves significantly. In figure 3 we show the expected value of $\mathrm{Om} 3$ determined using 500 simulated realizations of the BigBOSS experiment [37]. One clearly sees that $O m 3$ carries the potential to strongly discriminate between rival cosmological models with least a-priori assumptions being made about the early/late universe.

While $O m 3$ can be very useful for testing cosmological models with minimal a-priori assumptions, having reliable information from the early and late universe can help us to determine $h(z)$ and, knowing the latter, one can easily reconstruct the $\mathrm{Om}$ diagnostic. In figure 4 one realization of BigBOSS data together with expected future measurements of $H_{0}$ with $2 \%$ uncertainty is used to determine $O m(z)$. As we see from this figure, the $O m$ diagnostic provides a powerful means to discriminate between rival DE models. 


\section{CONCLUSIONS}

In this paper we introduce a new null diagnostic customized for reconstructing the properties of dark energy from BAO data. $O m 3$, as a 3 point diagnostic of dark energy, is closely related to the $O m$ diagnostic and follows the same general principles. Om3 is designed in a way that can be applied directly to BAO and supernovae data in order to falsify concordance cosmology. This is done in a completely non-parametric way. The importance of $O m 3$ lies in the fact that it does not rely on a knowledge of $\Omega_{0 m}, H_{0}$, or the distance to the last scattering surface, which permits $O m 3$ to falsify the $\Lambda \mathrm{CDM}$ model independently of these quantities. $\mathrm{Om} 3$ shares a common property with its cousin the $\mathrm{Om}$ diagnostic, namely, the values of both $O m(z)$ and $O m 3(z)$ stay pegged (at unity for $O m 3(z)$ and $\Omega_{0 m}$ for $O m(z)$ ) in an expanding concordance cosmology $(\Lambda \mathrm{CDM})$. This property of $\mathrm{Om} 3$ serves as a null test of the cosmological constant hypothesis, since, if observations do indicate that $O m 3$ evolves with redshift, then this would imply that $w \neq-1$ for the equation of state of dark energy. We have shown that current BAO and supernovae data are in agreement with standard $\Lambda \mathrm{CDM}$, however the uncertainties on $O m 3$ are still pretty high. This is mainly due to the quality of available BAO data (current BAO data alone cannot put tight constraints on the cosmological quantities [28]) but future experiments, such as BigBOSS, can lead to much tighter determinations of $\mathrm{Om} 3$. Independence of $\mathrm{Om} 3$ from any a-priori assumptions of the early universe as well as values of $H_{0}$ and $\Omega_{0 m}$ are important salient features of this null diagnostic of concordance cosmology $(\Lambda \mathrm{CDM})$.

Finally we would like to highlight the main differences between the two null diagnostics of $\Lambda \mathrm{CDM}-O m$ and $O m 3$. $O m$ is quite clearly a powerful null test of cosmological constant, and as it requires only a knowledge of $h(z)$, it seems straightforward to use $\mathrm{Om}$ together with parametric or non-parameteric methods of reconstruction. However deriving $h(z)$ directly from cosmological observables in a purely model independent and non-parameteric manner is not always an easy task. Though using supernovae data one can still derive $h(z)$ in a model independent manner [25], deriving $h(z)$ directly from large scale structure data seems to be more difficult [27]. In this paper we have shown that $O m 3$, in contrast to $\mathrm{Om}$, is specifically tailored to be applied to baryon acoustic oscillation data (directly through BAO observables) and that, in this case, $O m 3$ depends on a fewer number of cosmological observables than $O m$.

\section{Acknowledgments}

A.S. thanks Chris Blake for useful discussions and for providing us with the early WiggleZ data. A.S. acknowledge the Max Planck Society (MPG), the Korea Ministry of Education, Science and Technology (MEST), GyeongsangbukDo and Pohang City for the support of the Independent Junior Research Groups at the Asia Pacific Center for Theoretical Physics (APCTP). He also acknowledges the support of the World Class University grant R32-2009000-10130-0 through the National Research Foundation, Ministry of Education, Science and Technology of Korea. A.A.S. acknowledges RESCEU hospitality as a visiting professor. He was also partially supported by the grant RFBR 11-02-00643 and by the Scientific Programme "Astronomy" of the Russian Academy of Sciences.

[1] S. J. Perlmutter, et al., Nature 391, 51 (1998); A. G. Riess, et al., Astron. J. 116, 1009 (1998) arXiv:astro-ph/9805201; S. J. Perlmutter, et al., Astroph. J. 517, 565 (1999) arXiv:astro-ph/9812133; J. L. Tonry, et al., Astroph. J. 594, 1, (2003) arXiv:astro-ph/0305008; R. A. Knop, et al., Astrophys.J. 598, 102, (2003) arXiv:astro-ph/0309368; B. J. Barris, et al., Astrophys.J. 602, 571 (2004) arXiv:astro-ph/0310843; A. G. Riess, et al., Astrophys.J. 607, 665 (2005) arXiv:astro-ph/0402512. P. Astier et al., Astron. Astroph. 447, 31 (2005) arXiv:astro-ph/0510447. A. G. Riess, et al., arXiv:astro-ph/0611572. W. M. Wood-Vasey et al., arXiv:astro-ph/0701041 M. Kowalski et al., Astrophys. J. 686, 749 (2008). arXiv:0804.4142]. W. J. Percival et al., MNRAS 401, 2148 (2010) arXiv:0907.1660. E. Komatsu, et al., Astrophys.J.Suppl.192:18,2011, arXiv:1001.4538.

[2] N. Suzuki et al., Astrophys.J. 746, 85 (2012) arXiv:1105.3470.

[3] W. J. Percival et al., Mon. Not. Roy. Ast. Soc. 401, 2148 (2010) arXiv:0907.1660.

[4] C. Blake et al., Mon. Not. Roy. Ast. Soc. 415, 2892 (2011) arXiv:1105.2862.

[5] C. Blake et al., Mon. Not. Roy. Ast. Soc. 418, 1707 (2011) arXiv:1108.2653.

[6] F. Beutler et al., Mon. Not. Roy. Ast. Soc. 416, 3017 (2011) arXiv:1106.3366.

[7] A. Einstein, Sitz. Preuss. Akad. d. Wiss. Phys.-Math 142 (1917).

[8] V. Sahni and A. A. Starobinsky, Int. J. Mod. Phys. D 9, 373 (2000) arXiv:astro-ph/9904398. S M. Carroll, Living Rev.Rel. 4, 1 (2001) arXiv:astro-ph/0004075; P. J E. Peebles and B. Ratra, Rev. Mod. Phys. 75, 559 (2003) arXiv:astro-ph/0207347; T. Padmanabhan, Phys. Rep. 380, 235 (2003) arXiv:hep-th/0212290; V. Sahni, Lect. Notes Phys. 653, 141 (2004) arXiv:astro-ph/0403324]; arXiv:astro-ph/0502032; E. J. Copeland, M. Sami and S. Tsujikawa, Int. J. Mod. Phys. D 15, 1753 (2006) [arXiv:hep-th/0603057]; J. A. Frieman, M. S. Turner and D. Huterer, Ann. Rev. Astron. Astroph. 46, 385 (2008); R. Durrer and R. Maartens, "Dark Energy: Observational and Theoretical Approaches", 
ed. P Ruiz-Lapuente (Cambridge UP, 2010), pp. 48 - 91 arXiv:0811.4132; M. Li, X-D. Li, S. Wang, Y. Wang, Commun.Theor.Phys. 56, 525 (2011) arXiv:1103.5870; C. Clarkson, G. Ellis, J. Larena and O. Umeh, arXiv:1109.2484 S. Nojiri and S. D. Odintsov, Phys.Rept. 505, 59 (2011) [e-Print: arXiv:1011.0544]; T. Clifton, P. G. Ferreira, A. Padilla and C. Skordis, Phys.Rept. 5131 (2012) [e-Print: arXiv:1106.2476].

[9] V. Sahni and A. A. Starobinsky, Int. J. Mod. Phys. D 15, 2105 (2006) arXiv:astro-ph/0610026.

[10] V. Sahni, A. Shafieloo and A. A. Starobinsky, Phys. Rev. D 78, 103502 (2008).

[11] C. Zunckel and C. Clarkson, Phys. Rev. Lett 101, 181301 (2008).

[12] A. A. Starobinsky, JETP Lett. 68, 757 (1998) arXiv:astro-ph/9810431.

[13] D. Huterer and M. S. Turner, Phys. Rev. D 60, 081301 (1999) [arXiv:astro-ph/9808133].

[14] T. Nakamura and T. Chiba, Mon. Not. Roy. Ast. Soc. 306, 696 (1999) arXiv:astro-ph/9810447).

[15] T. D. Saini, S. Raychaudhury, V. Sahni and A. A. Starobinsky, Phys. Rev. Lett. 85, 1162 (2000) arXiv:astro-ph/9910231.

[16] T. Chiba and T. Nakamura, Phys. Rev. D 62, 121301(R) (2000) arXiv:astro-ph/0008175.

[17] R. Jiminez, L. Verde, T. Treu and D. Stern, Astroph. J. 593, 622 (2003), arXiv:astro-ph/0302560; J. Simon, L. Verde and R. Jiminez, Phys. Rev. D 71, 123001 (2005), arXiv:astro-ph/0412269; D. Stern, R. Jiminez, L. Verde, M. Kamionkowski and S.A. Stanford, JCAP 02, 008 (2010) arXiv:0907.3149.

[18] A. Sandage, Astroph. J. 136, 319 (1962); G. McVittie, Astroph. J. 136, 334 (1962).

[19] H-J. Seo and D.J. Eisenstein, Astroph. J. 598, 720 (2003) arXiv:astro-ph/0307460.

[20] E. Gaztanaga, A. Cabre and L. Hui, MNRAS 399, 1663 (2009) arXiv:0807.3551.

[21] T-J. Zhang and C. Ma, Adv.Astron. 2010, 184284 (2010) arXiv:1010.1307.

[22] C. Blake et al., Mon. Not. Roy. Ast. Soc. 418, 1725 (2011) arXiv:1108.2637.

[23] V. Sahni, T.D. Saini, A.A. Starobinsky and U. Alam, JETP Lett. 77, 201 (2003) arXiv:astro-ph/0201498.

[24] M. Arabsalmani and V. Sahni, Phys.Rev. D83, 043501 (2011) arXiv:1101.3436.

[25] A. Shafieloo, U. Alam, V. Sahni and A. A. Starobinsky, Mon. Not. Roy. Ast. Soc. 366, 1081 (2006) arXiv:astro-ph/0505329.

[26] A. Shafieloo, Mon. Not. Roy. Ast. Soc. 380, 1573 (2007) arXiv:astro-ph/0703034.

[27] A. Shafieloo and C. Clarkson, Phys.Rev. D81, 083537 (2010) arXiv:0911.4858.

[28] A. Shafieloo, arXiv:1204.1109.

[29] S. del Campo, R. Herrera and D. Pavon, Int.J.Mod.Phys. D 20, 561 (2011) arXiv:1103.5492.

[30] A.V. Pan and U. Alam, arXiv:1012.1591.

[31] M. Visser, Class. Quant. Grav. 21, 2603 (2004) arXiv:gr-qc/0309109.

[32] G. Efstathiou, Nuovo Cim. B122, 1423 (2007) arXiv:0712.1513.

[33] D. J. Eisenstein et al., Astrophys.J. 633, 560 (2005) arXiv:astro-ph/0501171.

[34] T. Chiba and T. Nakamura, Prog. Theor. Phys. 100, 1077 (1998) arXiv:astro-ph/9808022.

[35] T. Chiba and T. Nakamura, Prog. Theor. Phys. 118, 815 (2007) arXiv:0708.3877 [astro-ph]].

[36] D. J. Eisenstein et al., Astron.J. 142, 72, (2011) arXiv:1101.1529].

[37] D. J. Schlegel et al., BigBOSS: The Ground-Based Stage IV Dark Energy Experiment, arXiv:0904.0468

[38] http://funk.on.br/pau-brasil/main.html

[39] R. Laureijs et al., Euclid Assessment Study Report for the ESA Cosmic Visions, arXiv:0912.0914. 

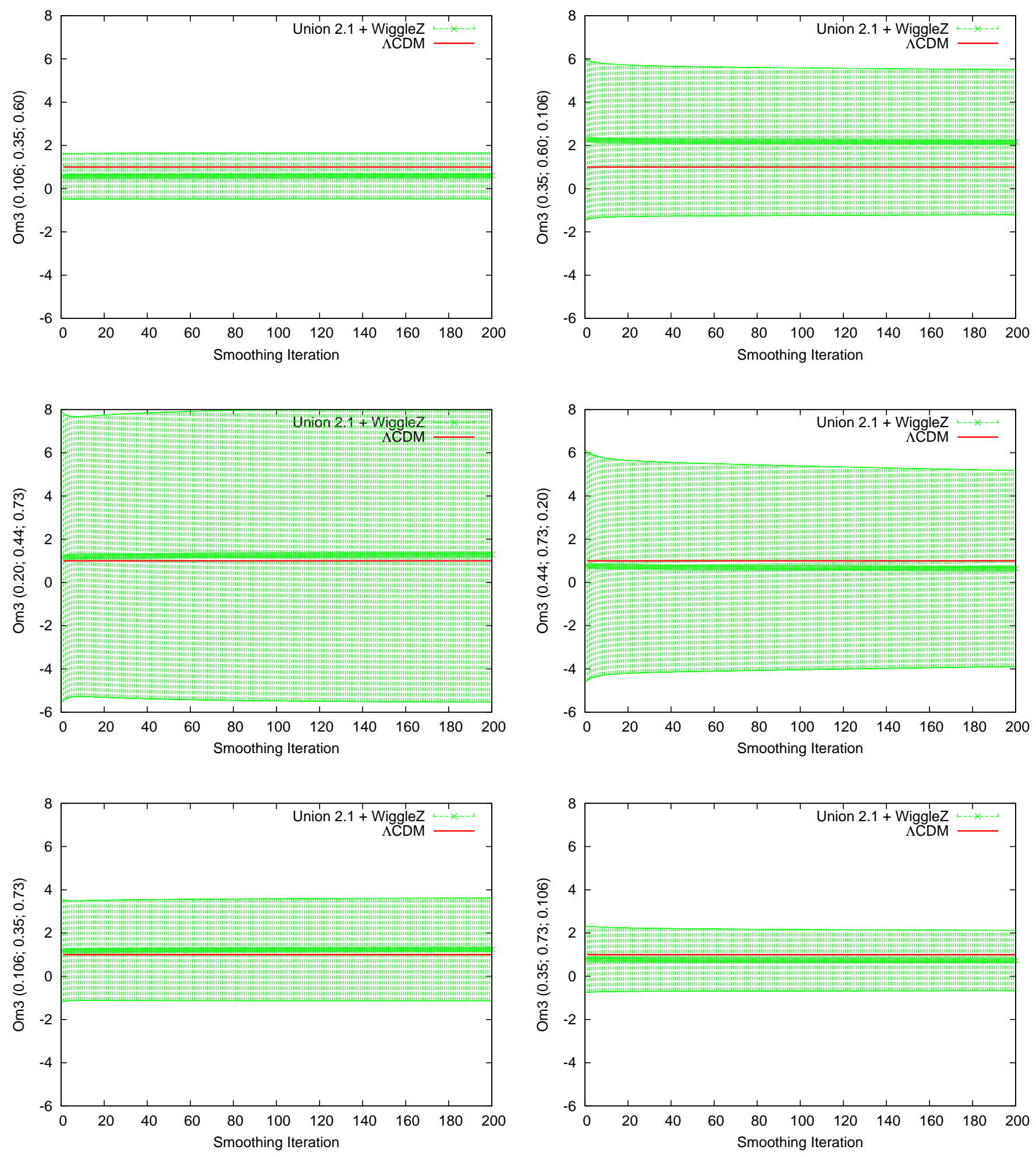

FIG. 2: Two variants of $O m 3$ determined using uncorrelated BAO measurements at three redshifts in conjunction with the luminosity distance reconstructed from Union 2.1 SNIa data. The redshift combinations Om3(0.106,0.35,0.60) \& Om3(0.35, 0.73, 0.106) shown in the top left and bottom right panels have the smallest error bars and therefore provide the tightest constraints on DE from current BAO+SNIa data. The large errorbars in the middle panels for Om3 are mainly due to the poor estimation of $d(z=0.44)$ (see Table 1$)$. The spatially flat $\Lambda$ CDM model is in good agreement with the data. Note that these results do not rely on a knowledge of $\Omega_{0 m}, H_{0}$, or the distance to the last scattering surface. 

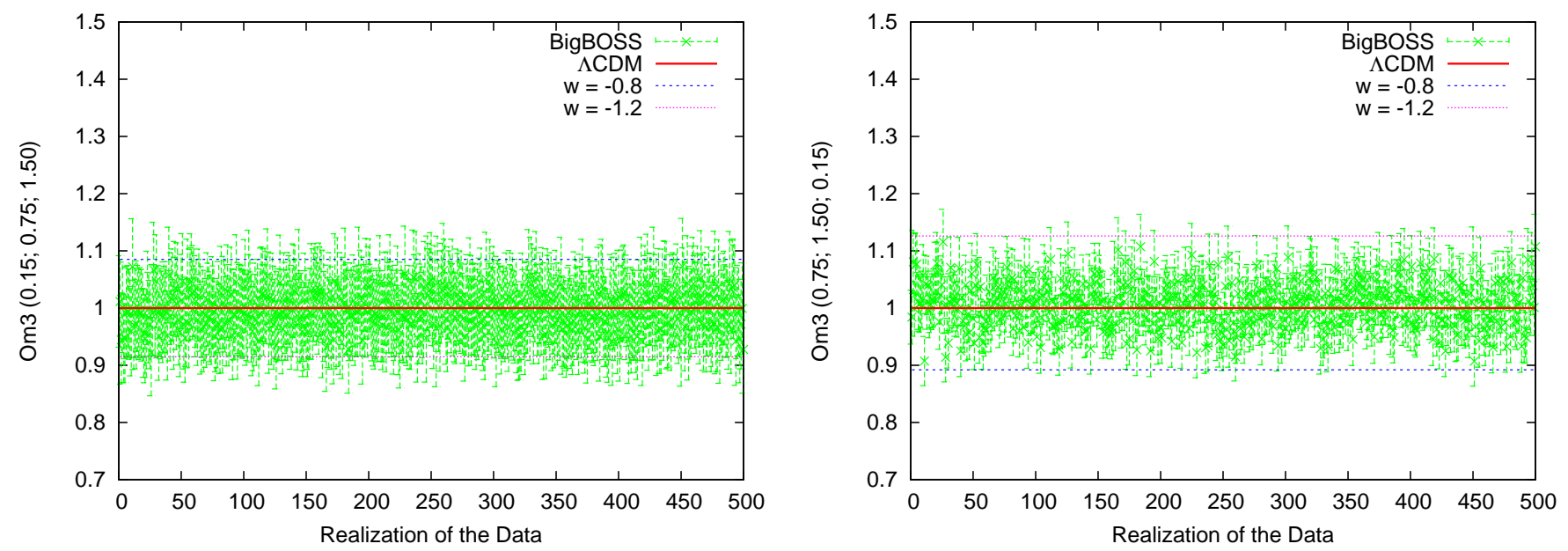

FIG. 3: Two variants of $O m 3$ derived using simulated realizations of the BigBOSS experiment assuming a fiducial $\Lambda$ CDM cosmology. Horizontal lines represent different dark energy models with (top-down) $w=-0.8, w=-1.0, w=-1.2 . \Omega_{0 m}=0.27$ is assumed for all models. Note that the determination of Om3 requires minimal cosmological assumptions since one does not require a background model to estimate $\Omega_{0 m}, H_{0}$, or the distance to the last scattering surface. Note also the difference in scale of the y-axis relative to figure 2 .

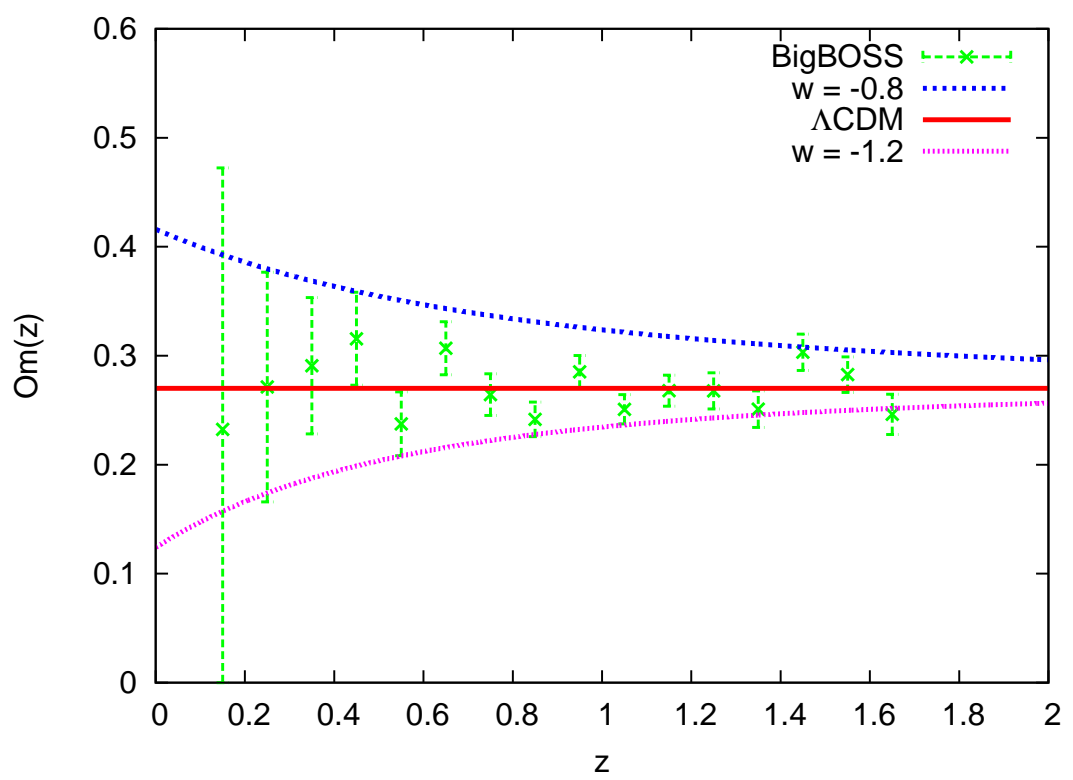

FIG. 4: The $O m$ diagnostic is reconstructed for a single simulated realization of the planned BigBOSS experiment assuming a fiducial $\Lambda \mathrm{CDM}$ cosmology. A determination of $h(z)$, and hence $O m(z)$, from future BAO experiments can clearly help distinguish between rival models of dark energy. Note that this determination is based on values of $H_{0}$ with $2 \%$ uncertainty expected by the time BigBOSS becomes operational. 